\title{
Die Lichtmessung unter Wasser mit Photoelementen: Ein Messverstärker mit linearer Anzeige
}

\author{
Von LÁSZLÓ KÁLMÁN \\ Eidg. Anstalt für Wasserversorgung, Abwasserreinigung und Gewässerschutz (EAWAG) an der \\ Eidg. Technischen Hochschule Zürich
}

Manuskript eingegangen am 1. Dezember 1972

\begin{abstract}
There are several alternative methods for the in situ measurement of light in water. Of the available methods the selenium photo element has been most widely used, largely because of its operational simplicity. The inherent deficiency of this technique, its nonlinear reading, could not be alleviated with the technology available at the time of the method's introduction. It is now possible to achieve perfect linearity with the aid of a modern operation amplifier, owing to the extremely low impedance of the photoelement input. The design of the apparatus is described in detail.
\end{abstract}

\section{Einleitung}

Mit den «Empfehlungen für die Durchführung von Strahlungsmessungen in Gewässern" von F. SAUBERER [7] hat die Technik der Licht- und Strahlungsmessung in der Limnologie einen wesentlichen Schritt vorwärts getan, und die Lichtmessung ist seither, namentlich im Zusammenhang mit der Messung der limnischen Primärproduktion, zu einem festen Bestandteil limnologischer Messtechnik geworden. Es ist aber doch unverkennbar, dass diese Messtechnik seit Sauberer mehr oder weniger stehengeblieben ist und dass trotz der riesigen Entwicklung der Elektronik das Angebot an käuflichen Messgeräten das ursprüngliche bescheidene Niveau nicht mehr verlassen hat. Wären die darin enthaltenen Geräte für die Bedürfnisse der limnischen Lichtmessung ausreichend, so wäre darin kein Mangel zu sehen. Leider ist dies nicht der Fall. Tatsache ist, dass das von Sauberer publizierte Lichtmessgerät bis zum heutigen Tage mit den gleichen systembedingten Nachteilen und Fehlern behaftet ist, auf die schon SAUBERER selber hingewiesen hat, die er aber mit den damals verfügbaren Mitteln nicht beheben konnte.

In der Zwischenzeit hat sich die Produktionsmessung als Routinemethode eingeführt, und dadurch sind die Ansprüche an die Strahlungs- bzw. Lichtmessung unter Wasser gestiegen. Die moderne Elektronik bietet ausgezeichnete Möglichkeiten, diese Technik zu verbessern. 


\section{Einige Möglichkeiten der Strahlungsmessung}

\subsection{Thermoelement, Thermosäule}

Die ankommende Strahlung wird durch eine Dunkelfläche absorbiert; die daraus resultierende Erwärmung wird durch Thermoelemente (Mollsche Thermosäule, Solarimeter, Sternpyranometer) gemessen. Diese Anordnung, welche in der Meteorologie und Limnologie routinemässig verwendet wird, gestattet die Absolutmessung der Gesamtstrahlung. Sie ist verhältnismässig wenig empfindlich und stellt an die Anzeigegeräte sehr hohe Anforderungen. Sie ist deshalb auf stationären terrestrischen Betrieb beschränkt. Eine Anwendung für Unterwassermessung ist prinzipiell möglich, aber technisch schwierig realisierbar und kostspielig, worauf übrigens Sauberer selber schon hingewiesen hat.

\subsection{Vakuumphotozelle}

In der Regeltechnik oft eingesetzt, sind Vakuumphotozellen heute gebräuchliche und preisgünstige Bauteile für die Lichtmessung. Sie werden mit einer Saugspannung betrieben; ihre Anzeige ist streng linear. Dagegen haben sie einen Dunkelstrom, der bei photometrischen Messungen stört und bei geringen Lichtstärken kompensiert werden muss. Die spektrale Empfindlichkeit der handelsüblichen Photozellen liegt zudem ungünstig für limnische Messung.

\subsection{Photomultiplier, Photovervielfacherröhre}

Durch Sekundäremission von Elektronen in einer Kaskadenschaltung von Hilfsanoden liefert der Multiplier, als Elektronenröhre ausgebildet, eine fast unbegrenzte Verstärkung des initialen Photostroms der Photokathode. Der Betrieb verlangt stabilisierte Hochspannung. Der Multiplier wird vor allem in empfindlichen Laborphotometern erfolgreich eingesetzt; für Lichtmessung unter Wasser sind einige Modelle beschrieben worden $[1,4]$. Eine allgemeine Anwendung hat sich daraus aber nicht ergeben, wohl auch deshalb, weil die extreme Empfindlichkeit des Multipliers nur für Spezialprobleme überhaupt ausgenützt werden kann, seine spektrale Empfindlichkeit aber nicht besser verteilt ist als bei allen andern photoelektrischen Sensoren. Photovervielfacherröhren sind zudem sehr leicht zerstörbar, sie erfordern für den Betrieb beträchtlichen apparativen Aufwand und sind unter sämtlichen photoelektrischen Sensoren die kostspieligsten. Trotzdem sind sie für Spezialzwecke unentbehrlich. Da sie ebenfalls Vakuumphotozellen sind, haben sie auch den erwähnten Dunkelstrom.

\subsection{Photowiderstände}

Die CdS-Photowiderstände sind heute als Regelglieder in Automatikschaltungen und vor allem als photographische Belichtungsmesser weitverbreitet. Als passive Schaltungsglieder altern sie sehr wenig. Ihr ausserordentlich grosser Messbereich (der Widerstand ändert sich negativ logarithmisch mit dem einfallenden Licht) wäre messtechnisch günstig, doch ist ihre spektrale Empfindlichkeit ungünstig, die Charakteristik nicht linear und zudem bei kleinen Lichtstärken nicht sehr konstant. Trotzdem können Photowiderstände in hochempfindlichen Messgeräten (z. B. Lichtmessung in grösserer Tiefe, Feststellung der Kompensationstiefe) erfolgreich eingesetzt werden $[6]$. 


\subsection{Photoelemente}

Während die Photoelemente aus der photographischen Lichtmessung weitgehend verschwunden sind, haben sie sich in der Limnologie aus den eingangs erwähnten Gründen bis heute gehalten. Ein weiterer Grund dafür ist darin zu suchen, dass die spektrale Empfindlichkeit der von Sauberer verwendeten Type besser ist als bei sämtlichen modernen Sensoren, gleichgültig, welcher Gruppe sie angehören.

Das Photoelement liefert elektrische Energie, welche der eingestrahlten Lichtenergie proportional ist. Es besteht aus einer metallischen Grundplatte, auf der (bei der Selenzelle) eine Schicht aus kristallinem Selen aufgebracht ist ( $p$-Leiter). Diese trägt eine durchsichtige, aufgedampfte Schicht von halbleitendem Kadmiumselenid ( $n$-Leiter), welche durch eine gut leitende durchsichtige Deckschicht aus Kadmiumoxid abgeschlossen ist. Der Strom wird an der Grundplatte und am Rand der Deckschicht abgenommen.

\subsubsection{Spektrale Empfindlichkeit}

Die spektrale Empfindlichkeit der Photoelemente variiert gemäss ihrer Bauart bzw. den verwendeten photosensitiven Materialien. Für limnologische Messungen eignet sich vor allem das Selenelement Typ N von Falkenthal \& Presser (Nürtingen, BRD), das der Augenempfindlichkeit am nächsten kommt und das den für die Photosynthese wichtigen Spektralbereich von 400 bis $700 \mathrm{~nm}$ gut einschliesst (Abb. 1).

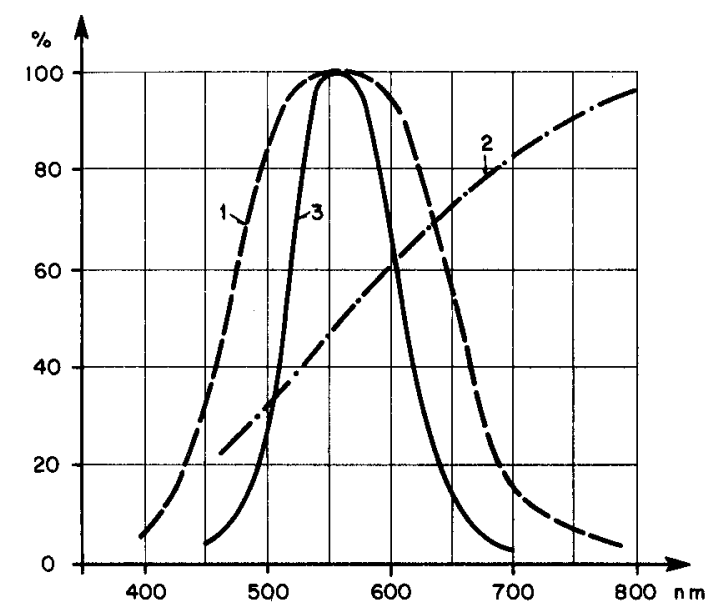

Abb. 1. Spektrale Empfindlichkeit von Photoelementen. 1. Selenelement Typ N von Falkenthal \& Presser (Nürtingen, BRD) ; 2. Siliziumelement; 3. Augenempfindlichkeit. Werte nach [7].

\subsubsection{Elektrische Charakteristik der Photoelemente}

Gemäss Abb. 2 ist die Leerlaufspannung, d.h. die Spannung zwischen den beiden Elektroden des unbelasteten Systems, etwa proportional dem Logarithmus der Beleuchtungsstärke. Da die Messung der Leerlaufspannung im Feldbetrieb aber nicht völlig leistungslos erfolgen kann, ist dieser Parameter messtechnisch uninteressant. 
Wird das System kurzgeschlossen, so fliesst zwischen den Elektroden ein Strom, der bis zu einer (typenabhängigen) oberen Grenze streng proportional zur Beleuchtungsstärke ist (Abb. 2).

Um eine vorzeitige Alterung der Photozelle auszuschliessen, vermeidet man Messungen in der Nähe dieser Proportionalitätsgrenze durch Zuschalten neutraler Graufilter.

\section{Die Messung des Photostroms und der Einfluss des Widerstands des Anzeigeinstruments}

Bei der praktischen Messung, welche mit den herkömmlichen Mitteln nicht unter Kurzschlussbedingungen ausgeführt werden kann, stört der Aussenwiderstand des Messkreises, also der Widerstand des Anzeigeinstruments, die Anzeige durch nichtlineare Verzerrung. Der Fehler steigt innerhalb des gleichen Messbereichs proportional zur Lichtintensität (Abb. 2). Bei Umschalten auf empfindlichere Messbereiche wird dagegen der relative Fehler entsprechend grösser. In besonderen Fällen lässt sich diesem Umstand durch nichtlineare Skalen oder Korrekturdiagramme begegnen. Dabei tritt aber die Schwierigkeit auf, dass jedes Photoelement und jeder Messbereich, ferner jede Spektralfilterkombination eine individuelle Eichung benötigt. In der Praxis lässt sich ein solches empirisches Verfahren kaum realisieren.

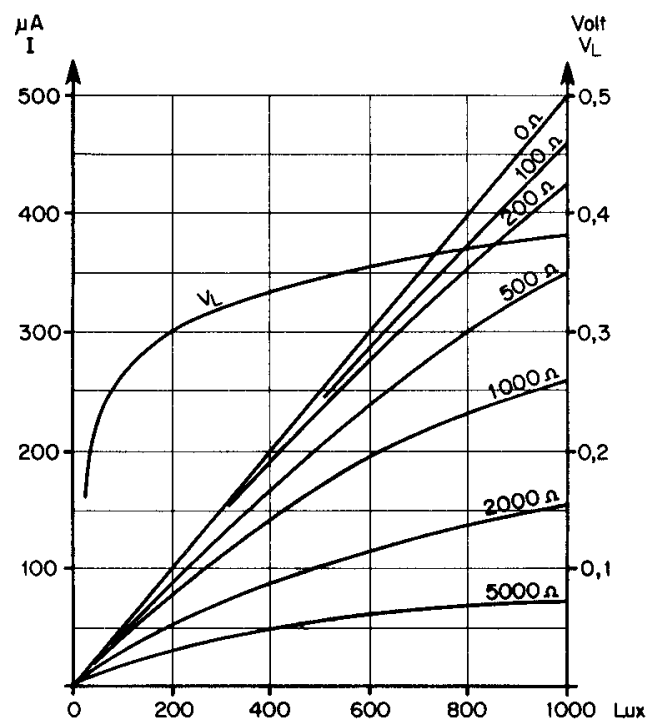

Abb. 2. Leerlaufspannung $V_{L}$ und Photostrom $I$ bei verschiedenen Aussenwiderständen in Funktion der Beleuchtungsstärke.

\subsection{Stromkompensationsmethoden}

Die Potentialdifferenz am Photoelement kann unter Verwendung eines Galvanometers mit Fremdstrom aus einer Batterie auf null kompensiert werden; gemessen wird der Kurzschlußstrom. Als interessanteste unter den vielen derartigen Schaltun- 
gen (publiziert durch GründLER [3]) sei die «Saugschaltung» nach MERZ und STANeK [8] erwähnt (Abb. 3).

Durch diese Schaltung lässt sich ohne eigentlichen Verstärker eine Stromverstärkung erzielen. Der Verstärkungsfaktor $V$ beträgt

$$
V=\frac{i_{i}}{i_{K}}=1+\frac{R_{2}}{R_{1}}
$$

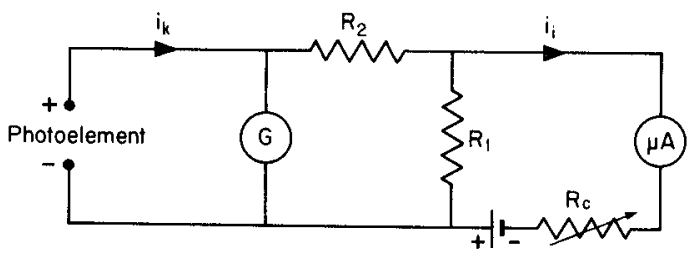

Abb. 3. "Saugschaltung» nach Merz und Stanek.

Durch Kompensation auf null am Galvanometer mit Hilfe des Verstellwiderstandes $R_{c}$ (Abb. 3) wird $i_{i}$ um ein Mehrfaches grösser als der Kurzschlußstrom $i_{K}$. Die Nullkompensation sowie das gleichzeitige Beobachten von zwei Instrumenten ist allerdings reichlich umständlich und bei wechselnder Lichtstärke sogar fast unmöglich.

\subsection{Transistorverstärker}

Die Verwendung von Transistorverstärkern brachte zunächst eine willkommene Erhöhung der Empfindlichkeit des Systems. Ohne komplizierte Schaltungen blieb aber die Konstanz der Verstärkung, die Linerarität der Anzeige sowie die Stabilität des Nullpunkts unbefriedigend.

Erst die Operationsverstärker, heute in integrierter Technik ausgeführt, brachten dank ihrer grossen Verstärkung einen wesentlichen Fortschritt.

\subsection{Operationsverstärker mit minimalem Eingangswiderstand}

Während die üblichen Operationsverstärkerschaltungen einen beträchtlichen Eingangswiderstand besitzen, zeichnet sich die nachfolgende Schaltung durch einen Eingangswiderstand von Null aus, wie aus der Prinzipschaltung (Abb. 4) ersichtlich ist.

Bei einem idealen Operationsverstärker ist der Verstärkungsfaktor

$$
V=\frac{i_{i}}{i_{K}}=1+\frac{R_{2}}{R_{1}}
$$

ferner Eingangswiderstand $r_{i}=0$ und

Ausgangswiderstand $r_{a}=\infty$.

Wenn $R_{1}=\infty$ oder $R_{2}=0$, ist $V=1$.

Der Verstärkungsfaktor ist somit nur vom Verhältnis zweier Widerstände abhängig. Er ist dagegen unabhängig von der Speisespannung und vom Verstärkungsfaktor des Operationsverstärkers; der letztere muss aber hoch sein. Durch diese Bedingungen ist eine sehr gute Stabilität des Instruments gewährleistet. 

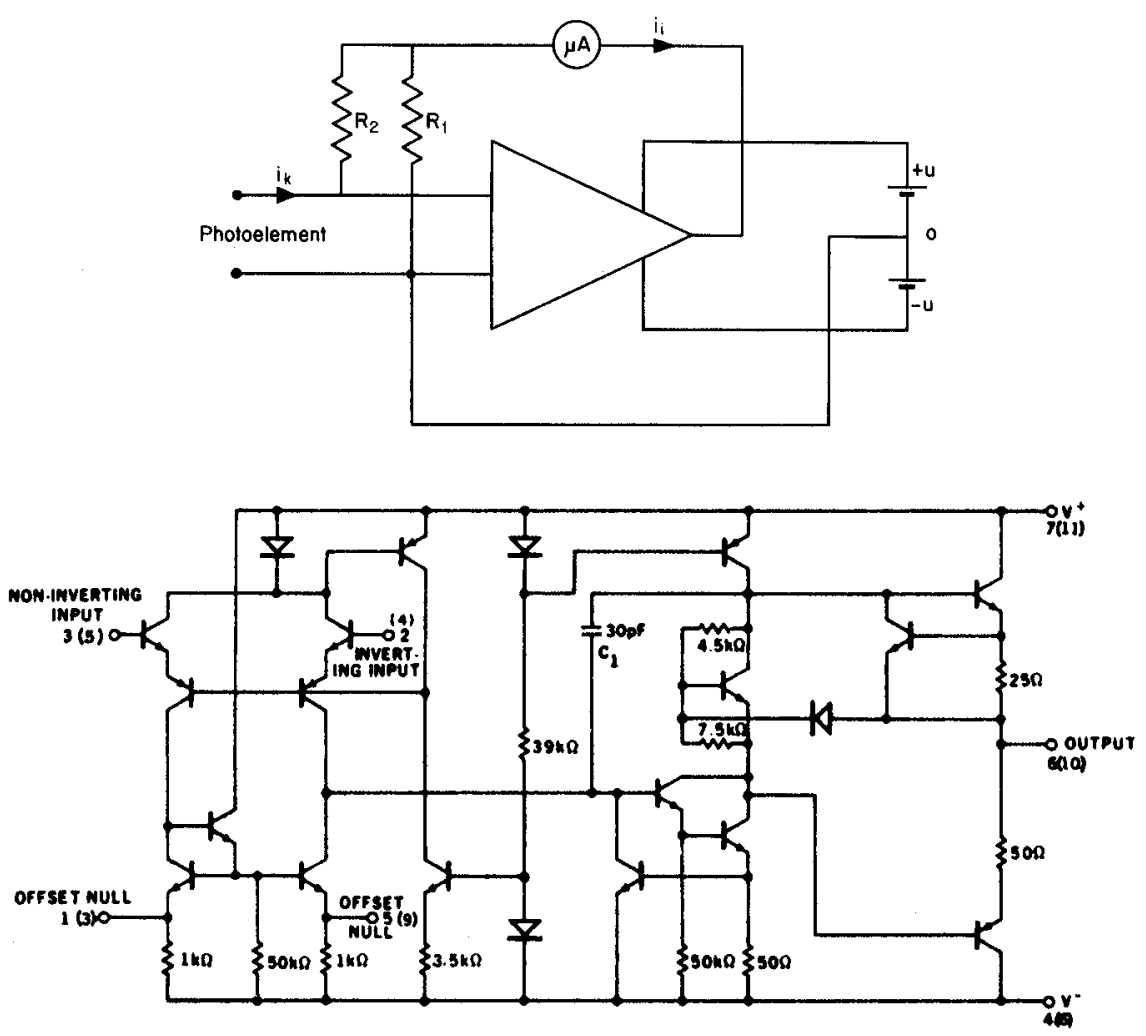

Abb. 4. a) Operationsverstärker, Prinzipschaltung. b) Schaltbild des Operationsverstärkers "Amelco Semiconductor 741".

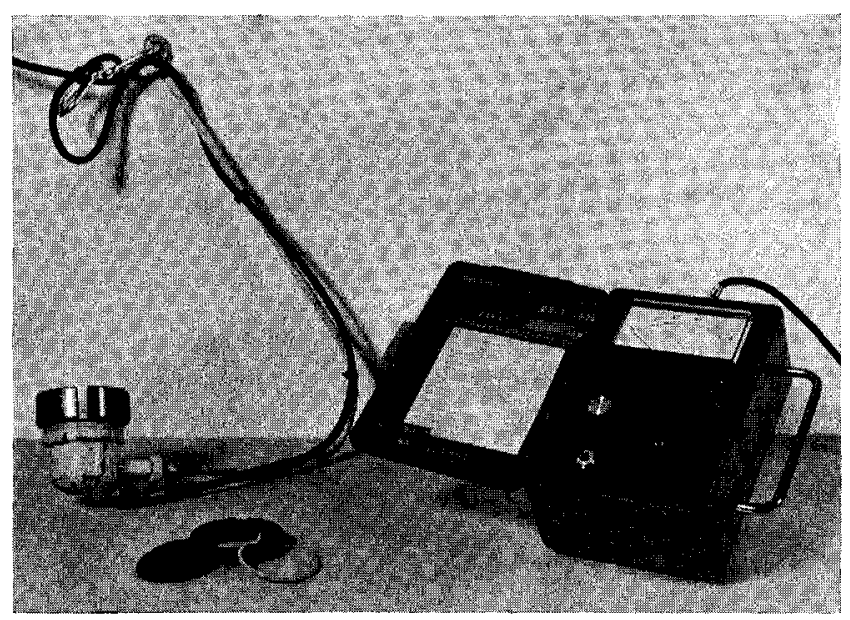

Abb. 5. Bild des Geräts. 


\section{Das Messgerät}

Das Gerät wurde nach der in Abb. 6 wiedergegebenen Schaltung an der EAWAG gebaut und bei limnologischen Messungen auf verschiedenen Seen erprobt (Abb. 5).

Der Messbereich reicht von 2,5 $\mu \mathrm{A}$ Vollausschlag (empfindlichste Stufe) bis $500 \mu \mathrm{A}$ (Schalter $\mathrm{S}_{\mathbf{1}}$ ). In der Stellung "Null» wird mit dem Potentiometer $\mathrm{R}_{8}$ der Nullpunkt korrigiert. Diese Einstellung bleibt für längere Zeit stabil; sie kann jederzeit nachgeprüft werden. Die Schalterstellung «Batterie» erlaubt die Kontrolle der Kondition der Batterien. Es werden handelsübliche Transistorbatterien $9 \mathrm{~V}$ verwendet; dank dem geringen Arbeitsstrom von $1,5 \mathrm{~mA}$ halten sie sehr lange. Die Kondensatoren $\mathrm{C}_{1}$ und $\mathrm{C}_{2}$,

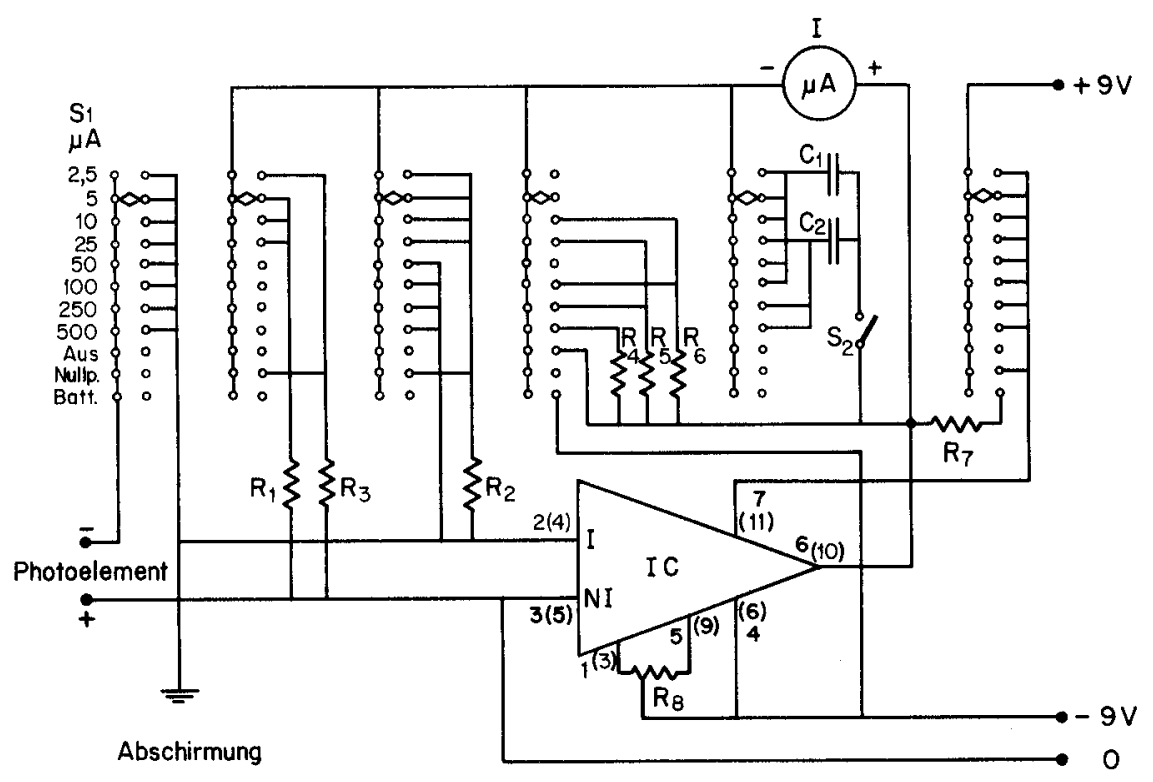

Abb. 6. Schaltschema.

Stückliste:

$S_{1}$ Stufenschalter $6 \times 11$

$\mathrm{S}_{2}$ Dämpfungsschalter

I Messinstrument $50 \mu \mathrm{A} / 2500 \Omega$

Widerstände:

$\mathrm{R}_{1}=1111 \Omega$

$R_{2}=10000 \Omega$

$\mathrm{R}_{3}=526 \Omega$

$\mathrm{R}_{4}=278 \Omega$

$\mathrm{R}_{5}=625 \Omega$

$\mathrm{R}_{6}=2500 \Omega$

$\mathrm{R}_{7}=400 \mathrm{k} \Omega$

$\mathrm{R}_{8}=20 \mathrm{k} \Omega$

Kondensatoren:

$\mathrm{C}_{\mathbf{1}}=1000 \mu \mathrm{F}$

$\mathrm{C}_{2}=5000 \mu \mathrm{F}$

IC Operationsverstärker Typ $741 \mathrm{C}$ Amelco oder Fairchild (USA) 
welche mit dem Schalter $\mathrm{S}_{2}$ wahlweise zugeschaltet werden können, gestatten eine Dämpfung des Galvanometerausschlags, wenn infolge von Wellen die Lichtstärke am Sensor zu stark schwankt. Da eine völlig glatte Seeoberfläche sozusagen nie vorkommt, ist eine solche Dämpfung notwendig. Ihre Zeitkonstante beträgt 1,3 bzw. 2,6 sec.

Wegen seiner hohen Verstärkung ist das Instrument ziemlich empfindlich auf Fremdfelder. Da solche insbesondere auf Arbeitsbooten reichlich vorhanden sind, vor allem wegen der mit Gleichstrommotoren betriebenen Arbeitsmaschinen, ist eine wirksame Abschirmung unbedingt erforderlich.
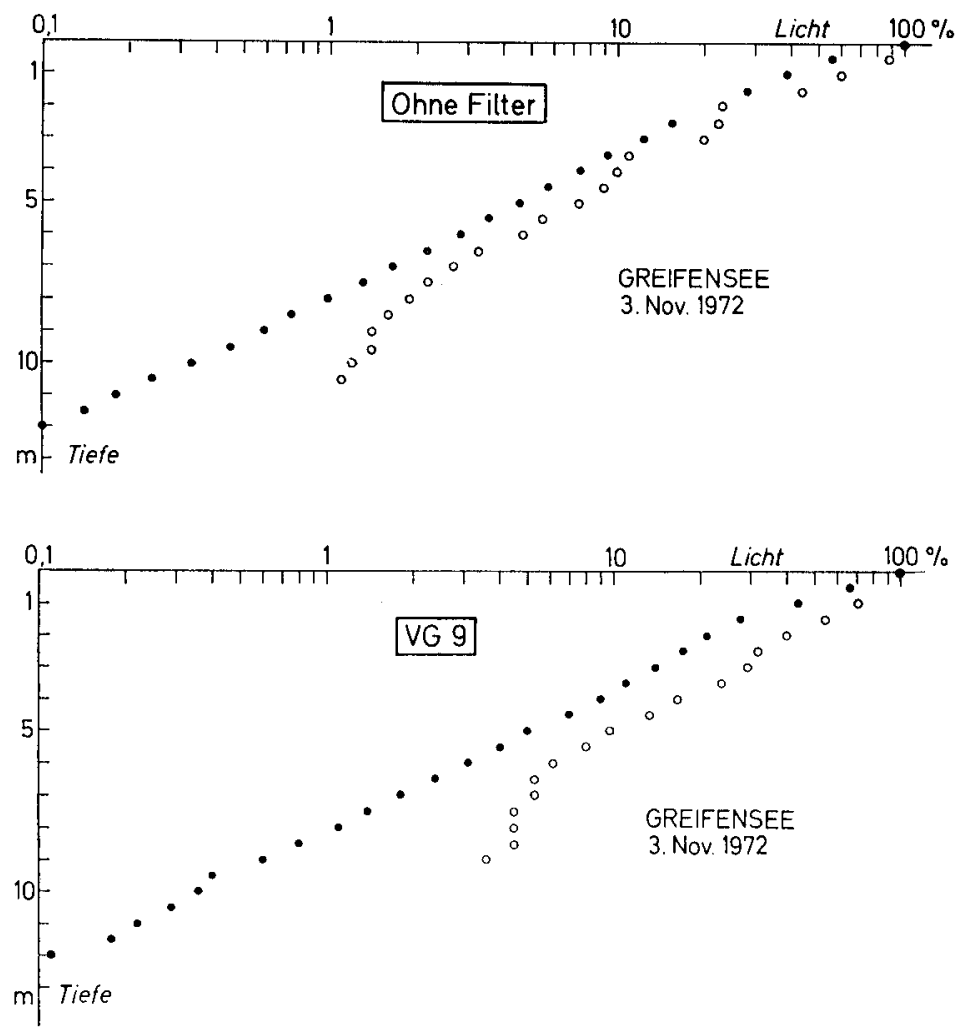

Abb. 7. Lichtmessungen (Greifensee, 3. November 1972):

- - mit Operationsverstärkergerät

- o o mit Galvanometer

In der vorliegenden Form erlaubt die Schaltung wegen der vorhandenen Offsetspannung nur den Eingang hochohmiger Spannungsquellen, was für Photoelemente zutrifft. Allfälliger Kurzschluss des Sensors wird spätestens bei der Nullpunktkontrolle angezeigt. Mit einer Kompensation der Offsetspannung könnte dieses Messprinzip auch noch für andere Aufgaben verwendet werden. 


\section{Anwendungsbeispiel}

In Abb. 7 sind zwei Beispiele von Lichtmessungen dargestellt, ausgeführt mit dem beschriebenen Operationsverstärkergerät und mit einem im Handel als Ausrüstung von Unterwasserphotometern angebotenen Drehspulgalvanometer (Vollausschlag bei $75 \mu \mathrm{A}$ ). Die Messungen wurden unter günstigen Bedingungen ausgeführt (Greifensee, 3. November 1972, Strahlung diffus infolge leichten Nebels, Wassermasse über die ganze Messtiefe hinweg nach vorangegangener Durchmischung wieder in Ruhe).

Direktmessung mit Galvanometer: Der bei hoher Lichtstärke auftretende negative Fehler macht sich sehr deutlich bemerkbar; beim Umschalten auf einen grösseren Messbereich des Instruments (verschiedene Shunts) tritt er jedesmal erneut auf. (Diese letztere Erscheinung sollte mit entsprechenden Seriewiderständen, mit denen der Gesamtwiderstand des Systems über sämtliche Empfindlichkeitsbereiche hinweg konstant zu halten wäre, eliminiert werden).

Messung mit Operationsverstärker: Weitgehend exponentielle Zunahme der Extinktion mit der Messtiefe. Die positive Abweichung von der Geraden in der obersten Wasserschicht ist auf höhere Transparenz (Sedimentation des Planktons infolge des windstillen Wetters) zurückzuführen. Dank der hohen Verstärkung ist das Gerät um 1,5 bis 2 Dekaden empfindlicher als das reine Galvanometer.

\section{Zusammenfassung}

Nach einer kurzen Aufzählung der technischen Möglichkeiten zur Unterwassermessung des Lichts in Gewässern wird das heute gebräuchliche Messverfahren mit Selenphotoelementen beschrieben. Dieses liefert nur bei niederen Lichtstärken lineare Anzeigewerte; bei höheren Lichtstärken wird die Anzeige ungenau. Beim Umschalten auf empfindlichere Messbereiche wird die relative Abweichung entsprechend grösser. Dieser systembedingte Fehler wird durch eine neue Messverstärkerschaltung (Operationsverstärker in integrierter Schaltungstechnik) vermieden. Die Konstruktion wird im Detail beschrieben.

Ich möchte erwähnen, dass ohne die wertvollen Ratschläge und kritische Mitarbeit von Prof. Dr. H. Ambühl sowie durch die Anregungen von Dr. R. Gächter diese Arbeit kaum entstanden wäre. Im weiteren möchte ich Herrn dipl. Chem. E. Szabó für die Durchführung und Auswertung der Messungen auf dem See, Fräulein Colette Kühn für die Übersetzung des Résumé und Herrn Dr. P. V. Roberts für die Übersetzung von Abstract und Summary danken.

\section{SUMMARY}

Following a brief account of the technical alternatives for the underwater measurement of light, the commonly used selenium photoelement technique is described. By its very nature this system provides a linear reading only at low light intensities. At higher intensities the reading is inexact. By switching to more sensitive measuring ranges the relative deviation becomes correspondingly larger. This deficiency can be avoided by means of a new amplifier circuit (integrated operation amplifier). Technical details are given.

\section{RÉSUMÉ}

Après une brève énumération des possibilités techniques de la mesure de la lumière sous l'eau, le procédé habituel, utilisant des éléments photosensibles au sélénium, est décrit. Celui-ci ne fournit des valeurs linéaires, pour des raisons dûes au système, qu'en présence de faibles intensités lumi- 
neuses. En présence d'intensités plus hautes, la valeur indiquée devient imprécise. En passant à des échelles plus sensibles, l'erreur relative devient proportionellement plus grande. Cette erreur est éliminée par un nouveau couplage d'amplificateur de mesure (amplificateur d'opération en circuits intégrés). Les indications techniques sont données en détail.

\section{LITERATURVERZEICHNIS}

[1] Acrefors, H., Ahnström, G., und Rosên, C.-G., Construction and Pevformance of a Sensitive Light Meter for Undervater Use, Limnol. Oceanogr. 14/4, 613-617 (1969).

[2] Berger, W., Das Photoelement in der Messtechnik, Lichttechnik 8/1 (1956).

[3] Gründler, W., Messung von Kurzschlußströmen von Photoelementen, Arch. tech. Messen, J 390-2 (März 1960).

[4] Kampa, M., Underwater Daylight and Moonlight Measurements in the Easter North Atlantic, J. Mar. Biol. Ass. UK 50, 397-420 (1970).

[5] Kohlrausch, F., Praktische Physik, Bd. 1 und 2, 22. Aufl. (Stuttgart 1968).

[6] Rich, P. H., und Wetzel, R. G., A Simple, Sensitive Undervater Photometer, Limnol. Oceanogr. 14/4, 611-613 (1969).

[7] SaUberer, F., Empfehlungen für die Durchführung von Strahlungsmessungen in Gewässern, Mitt. Int. Ver. Limnol. 11 (1962).

[8] Stanek, J., Nachrichtentechnik 7, 423 (1957).

[9] Tietze, U., Halbleiter-Schaltungstechnik, 2. Aufl. (Springer-Verlag 1971).

Anschrift des Verfassers: Dipl. Ing. L. Kálmán, Rautihalde 10, CH-8048 Zürich. 